\title{
Joubert Syndrome Presenting With Normal Pyramidal Decussation: A Case Report
}

\author{
Nam-Sik Kim, MD, Sung-Hee Park, MD, PhD
}

Department of Physical Medicine and Rehabilitation, Chonbuk National University Medical School, Jeonju, Korea

\begin{abstract}
Joubert syndrome (JS) is a rare genetic disorder characterized by a congenital malformation of the hindbrain, and accompanied by axonal decussation abnormalities affecting the corticospinal tract and the superior cerebellar peduncles. To the best of our knowledge, there are no reports of normal pyramidal decussation in JS. Here, we describe the case of an 18-year-old boy presenting midline-crossing corticospinal projections, which were considered normal corticospinal tract trajectories. Diffusion tensor imaging and motor evoked potential study analysis demonstrated the exclusive presence of decussating corticospinal projections in the patient. Based on these results, we suggest that JS might be associated with several, diverse corticospinal motor tract organization patterns.
\end{abstract}

Keywords Evoked potentials, Developmental disabilities, Joubert syndrome

\section{INTRODUCTION}

A congenital hindbrain malformation, Joubert syndrome (JS) is associated with axonal decussation abnormalities affecting the corticospinal tract (CST) and superior cerebellar peduncles [1]. A post-mortem neuropathological study has shown that pyramidal decussation is completely absent in patients with JS [2]. Recently, diffusion tensor imaging (DTI) analyses have also confirmed that the CST fails to decussate in JS [3]. Moreover, a neurophysiological study using transcranial magnetic stimulation (TMS) showed that individuals with JS display strong ipsilateral CST projections [4]. Taken together, these findings suggest that pyramidal tract fibers do not cross the midline in JS.

In this report, however, we present an exceptional case of JS characterized by only normally decussating CST projections.

\section{CASE REPORT}

An 18-year-old boy presented with a wide-based, unsteady gait. He had difficulty ambulating on uneven surfaces. Additionally, he presented noticeable oculomotor apraxia and mental retardation. His preferred hand was right, but he displayed severe clumsiness in both hands and had low hand strength. Mirror movements were not observed in either hand. Furthermore, the patient had

Received March 28, 2016; Accepted September 19, 2016

Corresponding author: Sung-Hee Park

Department of Physical Medicine and Rehabilitation, Chonbuk National University Medical School, 20 Geonji-ro, Deokjin-gu, Jeonju 54907, Korea. Tel: +82-63-250-2299, Fax: +82-63-254-4145, E-mail: shpark0130@jbnu.ac.kr ORCID: Nam-Sik Kim (http://orcid.org/0000-0003-0731-729X); Sung-Hee Park (http://orcid.org/0000-0002-4743-2551).

@ This is an open-access article distributed under the terms of the Creative Commons Attribution Non-Commercial License (http://creativecommons.org/ licenses/by-nc/4.0) which permits unrestricted noncommercial use, distribution, and reproduction in any medium, provided the original work is properly cited. Copyright $\odot 2017$ by Korean Academy of Rehabilitation Medicine 
displayed hypotonia during infancy, and presented a global developmental delay. Brain MRI demonstrated the presence of the molar tooth sign and vermian hypoplasia, typical of JS (Fig. 1A-1C).

We performed whole exome sequencing to look for the 19 genes associated with JS, namely: NPHP1, CEP290, AHI1, TMEM67 (MKS3), RPGRIP1L, CC2D2A, ARL13B, INPP5E, OFD1, TMEM216, KIF7, TCTN1, TCTN2, TMEM237, CEP41, TMEM138, C5orf42, TMEM231, and TCTN3. Genomic DNA was extracted from peripheral blood leukocytes using the Wizard Genomic DNA Purification Kit, as per the manufacturer's instructions (Promega, Madison, WI, USA). SureSelect Human All Exon V5 (Agilent Technologies, Santa Clara, CA, USA) was used for library preparation, and sequencing was done on the
Illumina NextSeq 500 platform (Illumina Inc., San Diego, CA, USA), generating $2 \times 150$ bp paired-end reads.

Results showed that the patient carried two compound-heterozygous mutations, a frameshift mutation (c.3557delA [p.Lys1186Argfs*22]) and a missense mutation (c.4277T>C [p.Ile1426Thr]) in the C5orf42 gene. Both these variants in C5orf42 were variants of uncertain significance, and have not been reported previously. Sanger sequencing confirmed these two variants in the C5orf42 gene (Fig. 1D). In the original family described by Joubert, Srour et al. [5] had reported that mutation of C5orf42 gene was the cause of JS, and the phenotype of this gene most closely resembled pure or classic JS.

DTI data were acquired using a Verio 3.0T system (Siemens, Erlangen, Germany) equipped with a 12-chan-

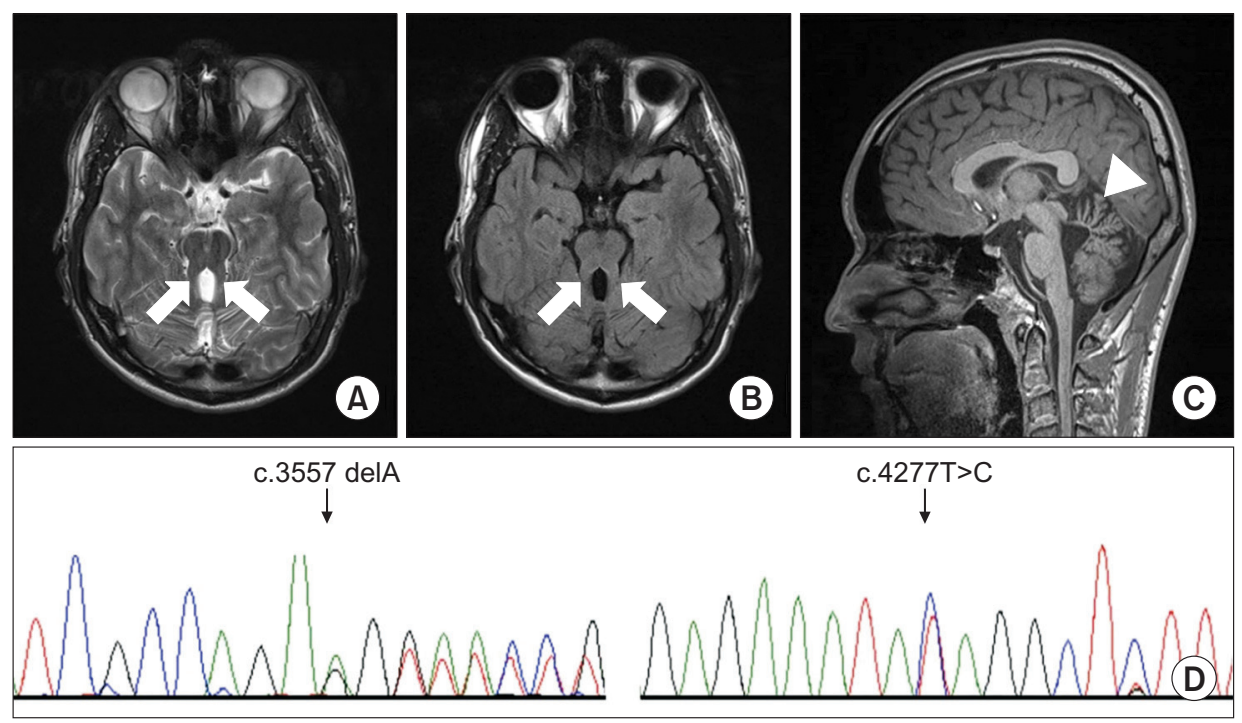

Fig. 1. Axial T2-weighted (A) and T2-weighted fluid-attenuated inversion recovery (B) brain magnetic resonance imaging scans at the pontomesencephalic junction show the molar tooth sign (arrow). The midsagittal T1-weighted image (C) shows hypoplasia of the cerebellar vermis (arrowhead). Sanger sequencing chromatograms verified c.3557delA and c.4277T>C in the C5orf42 gene (D). The black indicates a guanine, red indicates a thymine, blue indicates a cytosine, and green indicates an adenine.
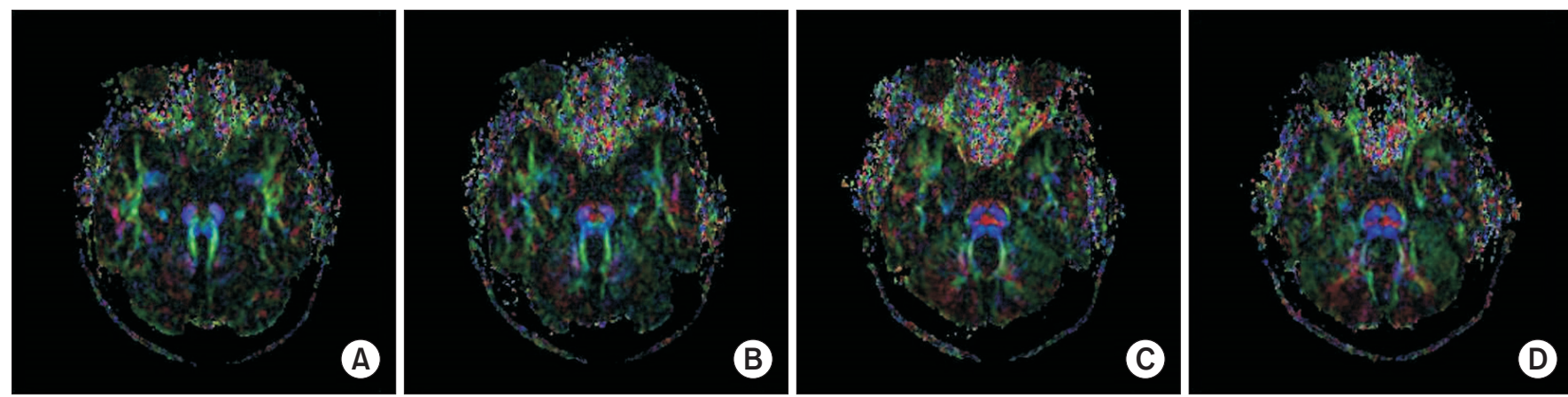

Fig. 2. Axial color-coded map at the level of the pontomesencephalic junction shows the absence of the midline 'focal red dot' which is interpreted as the absent decussation of the fiber tracts within the superior cerebellar peduncles (A). The transpontine fiber shows no abnormality at the basis pontis (B, C, D). The red indicates a predominant left-right (x-element), green indicates an anteroposterior (y-element), and blue indicates a superior-inferior (z-element) orientation of the anisotropic component of diffusion within each voxel. 
nel sensitivity encoding (SENSE) head coil for singleshot echo planar imaging. The imaging parameters used were: echo time $=93 \mathrm{~ms}$, repetition time $=7,900 \mathrm{~ms}$, field of view $=230 \mathrm{~mm} \times 230 \mathrm{~mm}$, sampling matrix size $=128 \times 128$ reconstructed with homodyne processing to $256 \times 256$, SENSE factor $=3$, EPI $=128$, and $b$-value $=1,000 \mathrm{~s} / \mathrm{mm}^{2}$. We acquired 47 contiguous, $3.0 \mathrm{~mm}$ thick slices parallel to the anterior commissure-posterior commissure line in 30 different diffusion directions.

DTI data analysis revealed absence of the midline 'focal red dot' at the level of the pontomesencephalic junction, representing the absent decussation of the superior cerebellar peduncle (Fig. 2A). At the basis pontis, the dorsal component of the transpontine fibers was found to project normally (Fig. 2B-2D). However, the deep cerebellar nuclei (dentate nuclei) appeared abnormally located more laterally (Fig. 3).

TMS was performed using a Magstim 200 stimulator (Magstim, Whitland, Wales, UK) equipped with a $65-\mathrm{mm}$ figure-eight coil. Motor evoked potentials (MEPs) from the first dorsal interosseous (FDI) muscle of both hands were recorded simultaneously. Stimulation intensity was set at $120 \%$ of the resting motor threshold. Each site was stimulated four times; the shortest latency and the average peak-to-peak (PTP) amplitudes were then measured from the recorded MEPs.

TMS of each hemisphere in the patient robustly elicited contralateral MEPs in the respective opposite hand, whereas no ipsilateral motor response could be induced
(Fig. 4A, 4B). Optimal stimulation sites for FDI muscles were identified about $5 \mathrm{~cm}$ lateral and $1 \mathrm{~cm}$ anterior to the vertex on both hemispheres. The excitatory threshold at the optimal stimulation was $77 \%$ in the left hemisphere and $87 \%$ in the right hemisphere. TMS of the right and left hemisphere elicited contralateral MEPs in the left hand (latency, 22.9 $\pm 0.4 \mathrm{~ms}$; PTP amplitude, 5.5 \pm 0.2 $\mathrm{mV}$ ) and contralateral MEPs in the right hand (latency, $22.1 \pm 0.3 \mathrm{~ms}$; PTP amplitude, $6.1 \pm 0.5 \mathrm{mV}$ ), respectively.

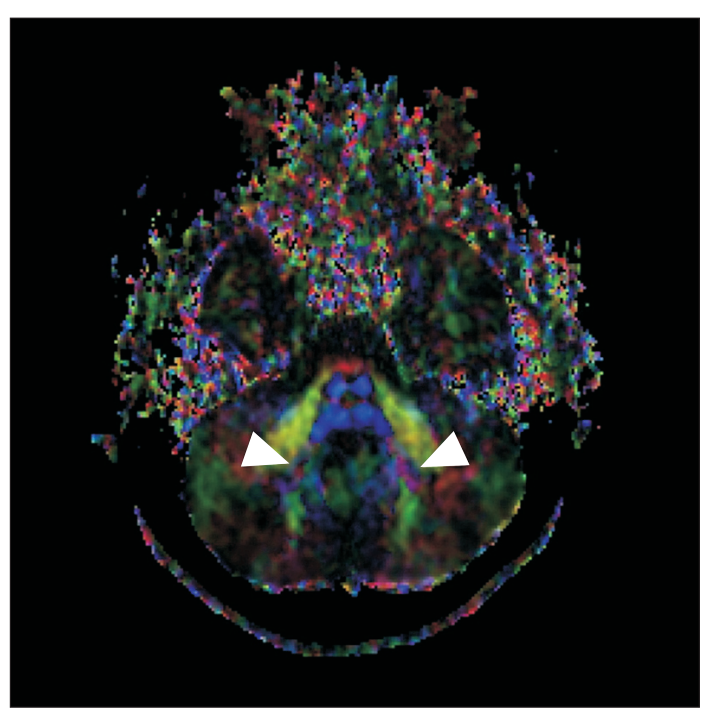

Fig. 3. Diffuse tensor imaging at the level of the dentate nuclei shows that the dentate nuclei is abnormally located more laterally (arrowheads).

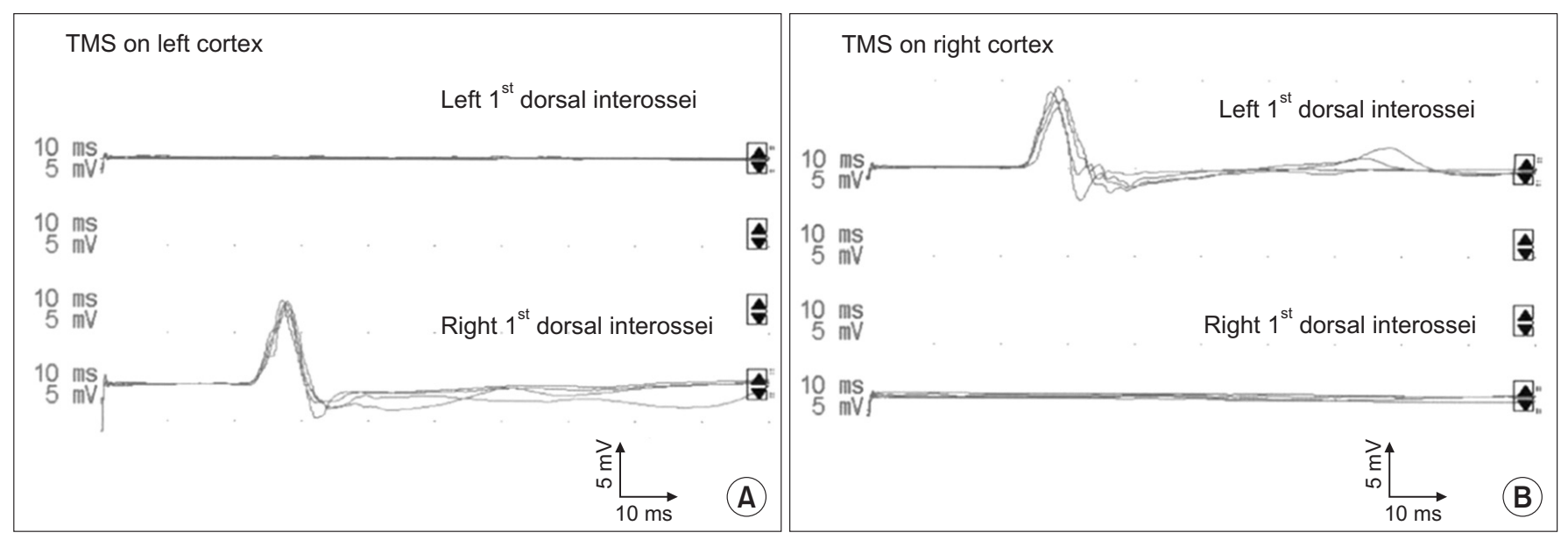

Fig. 4. Recording of the right and left 1st dorsal interossei motor evoked potentials with transcranial magnetic stimulation of left (A) and right (B) cortex shows contralateral responses only, suggesting crossed corticospinal projections from each hemisphere. TMS, transcranial magnetic stimulation. 


\section{DISCUSSION}

To the best of our knowledge, this study is the first to investigate the organization of the spinal motor system in JS using TMS. Our analysis demonstrated the presence of an exclusively decussated CST in one patient with JS, thus representing the first report of normal CST decussation in JS. Data on a possible presence of decussating fibers in corticospinal and superior cerebellar tracts in JS are scarce. Therefore, this condition is considered an exception to the Valsalva doctrine, which refers to the typical contralateral relationship of the brain to the body [6]. However, rudimentary decussation of the superior cerebellar peduncle fibers in JS has been reported in a few studies [2]. Among these, an fMRI study suggested the occurrence of a partial CST decussation in some JS cases [7]. Moreover, a recent neurophysiological investigation provided evidence of mildline-crossing corticospinal projections being present in some patients with JS [4]. However, these individuals concurrently showed ipsilateral corticospinal projections, and similar projections were also observed in the aforementioned fMRI study.

Therefore, until now, no fully normal pyramidal decussation has ever been reported in JS. This patient showed exclusively midline-crossing corticospinal projections, which were considered to be the normal CST trajectories. This indicates that the motor and sensory systems did not show a mismatch between the target of a specific motor output and the source of the corresponding sensory information in each cerebral hemisphere. Therefore, the patient could be functionally advantaged in the performance of voluntary limb movements.

Whole exome sequencing of the patient showed that he carried compound-heterozygous mutations in the C5orf42 gene. There could also be a possibility of normal corticospinal organization according to the genetic abnormality detected. Further studies are needed to elucidate the potential role of genes associated with JS.

We are aware that this study presents several limitations. Although the diagnosis of JS was based on clinical features as well as MRI findings of the patient, the use of molar tooth sign as a pathognomonic marker of JS has been previously criticized [8]. Furthermore, due to the current resolution of DTI, an accurate differentiation between a complete absence versus decreased volume of fibers is still not possible with this technique. Because of the technical limitations, we were unable to perform fiber tractography to show proper pyramidal decussation in the caudal medulla and crossing defects in the superior cerebellar peduncles.

In conclusion, this case report indicates that a variety of corticospinal motor organization patterns might characterize JS, in relation to the severity of hindbrain malformations and the extent of embryonic developmental defects affecting pyramidal decussation processes.

\section{CONFLICT OF INTEREST}

No potential conflict of interest relevant to this article was reported.

\section{REFERENCES}

1. Maria BL, Boltshauser E, Palmer SC, Tran TX. Clinical features and revised diagnostic criteria in Joubert syndrome. J Child Neurol 1999;14:583-90.

2. Yachnis AT, Rorke LB. Neuropathology of Joubert syndrome. J Child Neurol 1999;14:655-9.

3. Poretti A, Boltshauser E, Loenneker T, Valente EM, Brancati F, Il'yasov K, et al. Diffusion tensor imaging in Joubert syndrome. AJNR Am J Neuroradiol 2007; 28:1929-33.

4. Theoret H, Gleeson J, Pascual-Leone A. Neurophysiologic characterization of motor and sensory projections in Joubert syndrome. Clin Neurophysiol 2013; 124:2283-4.

5. Srour M, Schwartzentruber J, Hamdan FF, Ospina LH, Patry L, Labuda D, et al. Mutations in C5ORF42 cause Joubert syndrome in the French Canadian population. Am J Hum Genet 2012;90:693-700.

6. Schutta HS, Abu-Amero KK, Bosley TM. Exceptions to the Valsalva doctrine. Neurology 2010;74:329-35.

7. Parisi MA, Pinter JD, Glass IA, Field K, Maria BL, Chance PF, et al. Cerebral and cerebellar motor activation abnormalities in a subject with Joubert syndrome: functional magnetic resonance imaging (MRI) study. J Child Neurol 2004;19:214-8.

8. Dirik MA, Yis U, Dirik E. Molar tooth sign is not pathognomonic for Joubert syndrome. Pediatr Neurol 2013;49:515-6. 